\title{
Project Title: Colloid Genesis/Transport and Flow Pathway Alterations Resulting From Interactions of Reactive Waste Solutions and Hanford Vadose Zone Sediments
}

\author{
Principal Investigator: Jiamin Wan \\ Lawrence Berkeley National Laboratory \\ 1 Cyclotron Road, 70-108B \\ Berkeley, CA 94720 \\ 510-486-6004 \\ jmwan@lbl.gov \\ Co-investigator: $\quad$ Tetsu K. Tokunaga \\ Lawrence Berkeley National Laboratory \\ 1 Cyclotron Road, 70-108B \\ Berkeley, CA 94720 \\ 510-486-7176 \\ tktokunaga@lbl.gov
}

\section{Introduction}

The Hanford Site was one of the DOE's major nuclear weapons production sites from 1940 to 1989. Over time, 67 of the 149 single-shell tanks have leaked or are suspected of having leaked [1]. Contaminants such as ${ }^{99} \mathrm{Tc}$ and $\mathrm{U}$ have been found in elevated concentrations in the vadose zone and groundwater beneath the single shell tank farms [2]. In order to make decisions on remedial actions, numerous scientists have been investigating these problems through the DOE's Environment Management Science Program Hanford Vadose Zone Project. The U.S. Department of Energy has on-going projects at the Hanford site to monitor existing contaminant plumes in groundwaters, and to characterize the subsurface distribution of contaminants in tank farms. These efforts include the Tank Farm Vadose Zone Characterization Project and the 200 Area Remedial Action Project.

Site characterization activities have included collection of many subsurface samples exhibiting varying degrees of contamination and physical/chemical changes. These activities provide the basis for corrective and remedial action decisions, and identify scientific questions for resolution through research [3]. Although much has been learned concerning the waste migration, the combination of subsurface heterogeneity and limitations on field borehole drilling prevent comprehensive, high-resolution inventories of the various contaminants. While a number of laboratory-based studies have made considerable progress towards understanding reactions between tank waste solutions and Hanford sediments, the integration of laboratory and field understanding remains a challenge. Several factors contribute substantially to the problem of bridging the gap between field-based observations and many laboratory findings. Most laboratory studies, being focused on specific interactions, employ batch equilibration approaches that, in principle, occur somewhere within the contaminated region. However, such approaches by themselves can not generally identify where within a contaminant plume their isolated reactions are important. Linking such laboratory results to field data is made even more difficult when 
recalling that only very small fractions of contaminant plumes are typically sampled or otherwise characterized. Our research is directed at bridging this gap between field characterization and batchtype laboratory experiments.

\section{Methods and Materials}

We developed a laboratory column profiling method that provides measurements that have drect correspondence to field contaminant plumes. Besides directly integrating geochemical reactions and transport processes, the method permits direct sampling of pore solutions and sediments within a waste plume with high spatial and temporal resolution. Using this approach, we studied the Hanford SX tank waste leakage problem, and compared our laboratory results with geochemical data recently obtained by researchers from the field site characterization project.

Sediment: The sediment used for packing columns is the Hanford Formation "coarse sand", a glaciofluvial sediment, collected from 200 East Area of the Hanford site. The 200 Area is the principal waste management area at Hanford and houses high-level waste tanks. The Hanford formation comprises about $85 \%$ of the vadose zone of the site. The sand facies are mostly coarse to medium, and contain scattered well-rounded pebbles and cobbles, with some thin discontinuous silt lenses [4]. The major components are feldspar, quartz, and basaltic rock fragments [5,6]. The sediment used in this study contains $92.5 \%$ sand, $5.9 \%$ silt, and $1.7 \%$ clay. The median grain-size is $350 \mu \mathrm{m}$, and average

grain density is $2.77 \mathrm{Mg} \mathrm{m}^{-3}$. Calcium carbonate comprises $1.1 \%$ of the total mass. The $\mathrm{pH}$ of water extract was 8.4 (water to soil mass ratio $=1: 1$, at $21^{\circ} \mathrm{C}$ for 24 hours).

Simulated tank waste solution (TWS): The chemical composition of our simulated TWS is based on the historical analyses of the SX-111 tank waste. The reasons for choosing this solution are, firstly, because the reported composition is based on direct measurements. It is one of few available data sets from historical chemical analyses of tank supernatant solutions. Secondly, the data are well within the range of Agnew's [7, 8] estimates of SX tank supernatant solutions. Thirdly, the solution is stable at room temperature, and easy to handle in the laboratory. Major ions in our simulated TWS include 0.8 $\mathrm{M} \mathrm{Al}^{3+}, 8 \mathrm{M} \mathrm{Na}^{+}, 5 \mathrm{M} \mathrm{NO}_{3}^{-}$, and $\mathrm{pH}$ is 14 . All salts added were completely dissolved within hours of stirring at room temperature. The solution was filtered $(0.45 \mu \mathrm{m})$ before use. Radionuclides were not included in the simulated TWS because this study focused on effects of major chemical components.

Column and Column-sectioning Method: Each experiment was conducted by infusing simulated tank waste solution into a sediment column. Three different column lengths $(0.20,0.55$, and $2.05 \mathrm{~m})$ were used to experimentally simulate a waste plume at different stages. The experiments were conducted with the column temperature maintained at $70 \pm 1.0^{\circ} \mathrm{C}$. The simulated tank waste solution (TWS) was injected into the sediment column using a syringe pump, at a pore-water velocity of $100 \mathrm{~mm} \mathrm{~d}^{-1}$. When the effluent was just detected emerging at the outlet, injection was terminated and the column was immediately sectioned. Pore-solution samples were immediately vacuum extracted from each sediment segment. The solutions extracted from different sections were analyzed for $\mathrm{pH}$, electrical conductivity (EC) and major elemental composition. Sediments sampled from each segment were analyzed for their 
major element composition. The resulting spatially resolved chemical analyses permitted reconstruction of the evolution of the waste plume.

Chemical Analysis Methods: Measurements of $\mathrm{pH}$ were obtained using a non-glass $\mathrm{pH}$ probe (Sentron IntelliProbes). EC measurements were conducted on solutions that were 100-times diluted with distilled water. This level of dilution provided EC values that were linearly correlated with sums of major ion concentrations. For the major element compositions of the extracted pore liquids, a ICPOES (Thermo Jarell Ash Hi Res IRIS) was used. Since many of these liquid extracts contained precipitates (formed after the extraction), aqua regia, hydrofluoric acid, and boric acid were used to redissolve precipitates [9]. The sediment segments were analyzed using X-ray fluorescence (XRF).

\section{Results and discussion}

An example of the measured profiles of pore water $\mathrm{pH}$, salinity, and $\mathrm{OH}^{-}$(calculated from $\mathrm{pH}$ ) is presented in Figures 1. The ordinates in the figure are oriented to reflect the field geometry with downward migration of the contaminant. The pore water salinity is presented in the left panel in terms of normalized EC (relative to that of the initial TWS). Moving upward towards the TWS source from the uncontaminated displaced soil water, the region with rapidly increasing EC values indicates mixing of TWS (containing $8 \mathrm{M} \mathrm{NaNO}_{3}$ ) with the initial soil solution. The inflection point of an EC profile is used to locate the plume front. The plume front location agreed with calculations based on flow rate and the initial soil moisture content. This front separates the displaced soil water zone and neutralized TWS zone. The displaced zone contains primarily displaced initial soil water, and is characterized by its normalized EC being closed to zero and its $\mathrm{pH}$ being close to 8 . The displaced soil water zone exhibits increasing influences from mixing with neutralized TWS as the plume front is approached. 


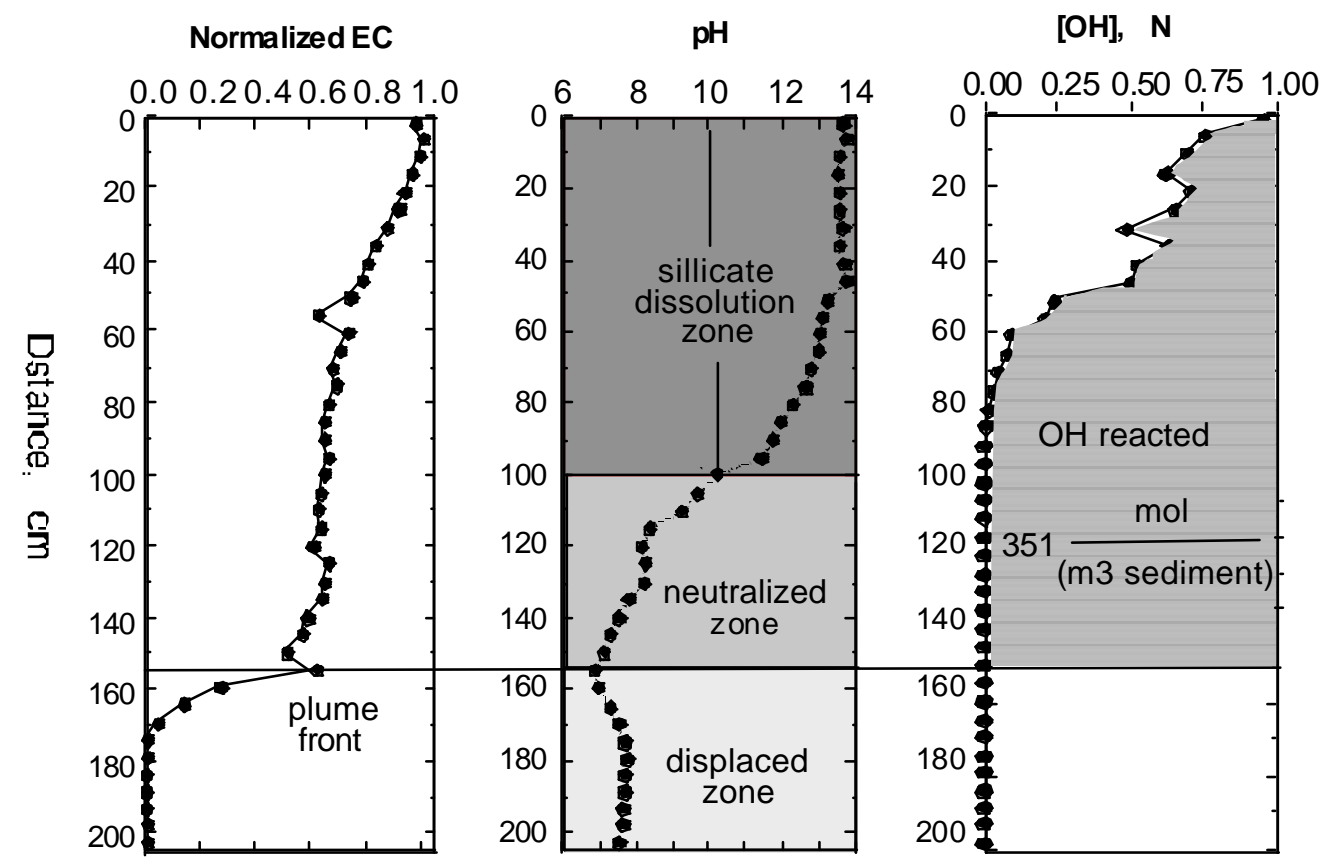

Figure 1. EC, $\mathrm{pH}$, and $\left[\mathrm{OH}^{-}\right]$profiles along the $2.05 \mathrm{~m}$ column.

The $\mathrm{pH}$ varied from 7 to 14 from the plume front to near the plume source (center panel in Figure 1). Because of this very wide range in $\mathrm{pH}$, and related wide range in overall geochemistry, two broad zones within the waste plume were defined: the neutralized zone and the silicate dissolution zone. The neutralized zone is taken from the plume front back upstream to a $\mathrm{pH}$ of 10 . This $\mathrm{pH}$ is assigned to the upper boundary of neutralized zone and the lower boundary of the silicate dissolution zone, and was chosen based on the relatively low solubility of silicates below this value. The high EC values of the neutralized zone indicate that the origin of the fluid is primarily the TWS. The neutralized zone $\mathrm{pH}$ values range from 6.3 to 10 , and the length of this zone grows in proportion to the length of the waste plume. The neutralized zones within the $0.2,0.55$, and $2.05 \mathrm{~m}$ columns are $32 \%, 36 \%$, and $37 \%$ of the total plume length, respectively.

Defined by $\mathrm{pH}$ values greater than 10, the silicate dissolution zone (SDZ) comprises the largest portion of the waste plume. Within this zone the $\mathrm{pH}$ monotonically rises up to 14, the value of the initial TWS, as the source is approached. Primary silicate dissolution and zeolite precipitation have been reported as the dominant reactions when Hanford REDOX TWS reacted with the sediment [10, 11]. The approximate $\mathrm{OH}^{-}$concentration profile was calculated based on $\mathrm{pH}$ data, and presented in the right panel of Figure 1. Integration of the area between the $\mathrm{OH}^{-}$profile and the $1 \mathrm{~N}$ (initial TWS [OH] $\mathrm{OH}^{-}$line, from the source position down to the plume front provides an estimate of the amount of $\mathrm{OH}^{-}$reacted per unit volume of sediment. From these column experiments, we have an estimate of 317 to 380 moles $\mathrm{OH}^{-}$reacted per cubic meter of sediment. 
A summary of the 3 columns of different lengths, with their different zones, is provided in Figure 2 Within the tested range, the silicate dissolution zone and neutralized zone both grow in approximately direct proportion to time and the volume of leaked TWS. The estimated amounts of $\mathrm{OH}^{-}$consumed per bulk volume of sediment from three columns are fairly similar.

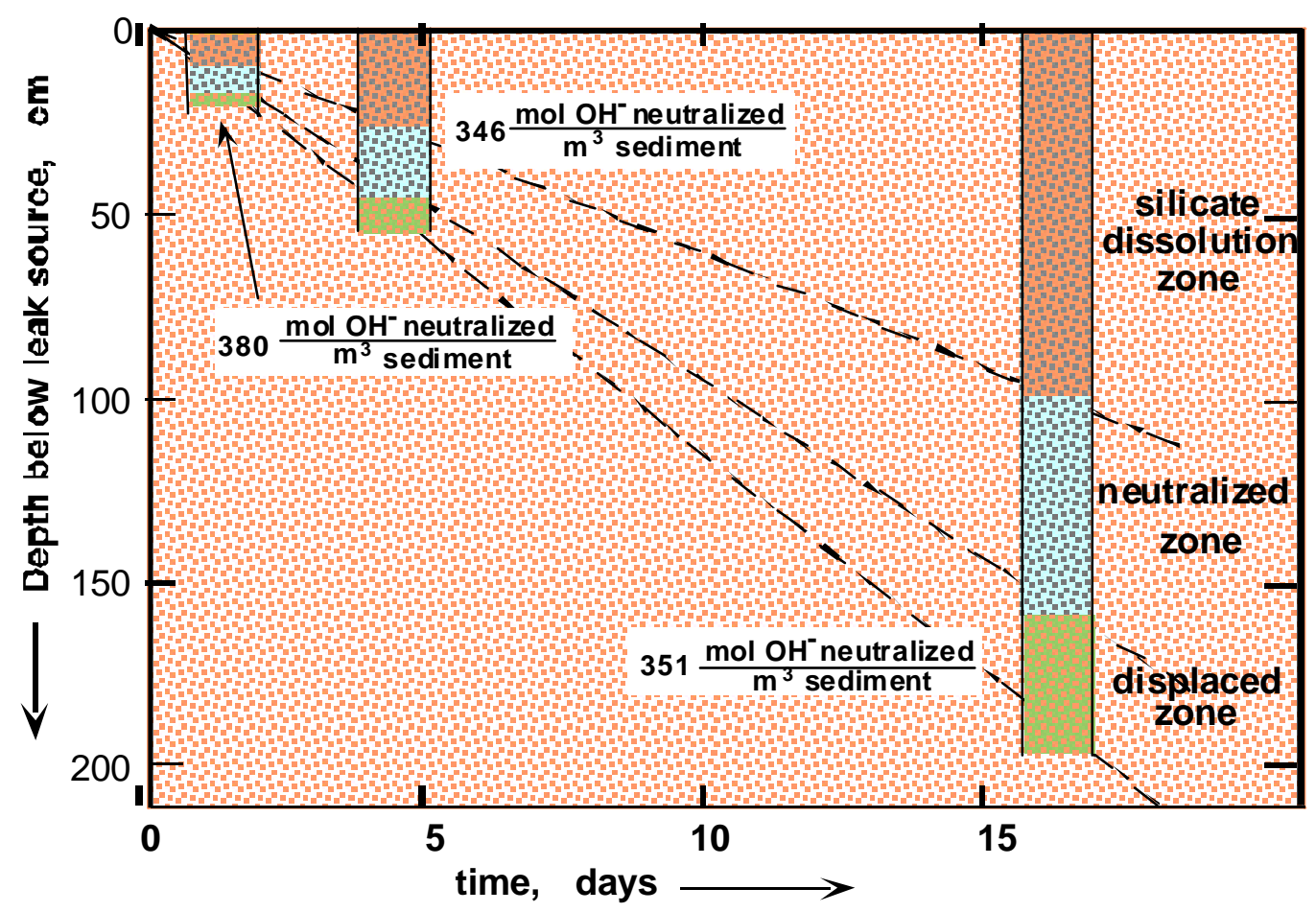

Figures 2. Summary and comparison of 3 experimental columns of different lengths, showing zones of silicate dissolution, $\mathrm{pH}$ neutralization, and displaced soil water. Also shown are estimates of $\mathrm{OH}^{-}$ neutralization from each column.

In order to understand the process of TWS $\mathrm{pH}$ neutralization, we studied the evolution of the waste plume solution chemistry, as well as evolution of the sediment's chemical and mineralogical composition. The samples of pore solutions extracted from individual column sections were analyzed for major elements to obtain the concentration profiles. Chemical analyses were also obtained on segments of sediments from which the solution samples were extracted. The secondary solid phases from individual segments of a sediment column are been identified using XRD. These profiles of elemental concentrations in the waste plume solutions and sediments, as well as the identified new phases provide validation of our thermodynamic calculations and a conceptual model of waste plume evolution. Some of the aforementioned work is still ongoing, and a final report will be submitted at the end of this FY. 
